\title{
Discussion on the Tone of Japanese
}

\author{
He Wang \\ School of Foreign Studies, Xi'an University, Xi'an, 710065
}

Key words: Tone, overview, type, rule

\begin{abstract}
From the current situation in the teaching and learning of Japanese tones, more and more attention has been paid to the tone of Japanese language. The overall level has been greatly improved no matter from the breadth and depth of the content of the study. Although lots of textbooks and dictionaries and other materials have been marked out the Japanese word tones for those Japanese learners, it did not provide concise and clear rules. With the increase of learning content, the diversity of tone change is more and more obvious, in which the vocabulary is increasing, and the structure of the article is more complicated. It is difficult to solve the practical problems that are found during learning by relying solely on the primary stage of rote learning and traditional learning methods. It is a problem that every Japanese learner has to solve in order to master the rules of tones correctly and improve the efficiency of learning. All the known language in the world, there are some words with higher or heavier pronunciations in their words. The traditional phonetics or phonology called these higher or heavier pronunciations as "stress". Stress in different languages has different forms of expression, and some are mainly reflected in the strength and weak. The tone of Japanese is expressed in high and low, and its pronunciation type has $n+1$. Different types of pronunciation have the corresponding pronunciation rules.
\end{abstract}

\section{Tone and type of tone}

The tone is the form of high and low syllable which is mainly determined by the pitch. The musical scale is also determined by the pitch, and it can be used to simulate the scale. Meanwhile, tone's learning can also help with their sense of music. However, it is worth to note that the pitch of the tone is relative, not absolute. The tonal movements of the tones are slippery and do not move as leaping from one scale to another. The levels of the tone are usually marked with a five-degree method: make a vertical line and divide into 5 degrees, the minimum level is 1 , and the maximum is level 5. Mandarin has four tones: Yinping Yangping, Shangsheng, and Qusheng.

Strength and weak type is reflected in English, high and low type is reflected in Japanese and Chinese. The tone is called "tone class", which is in accordance with the actual tone of pronunciation, and the same tone-values are classified into a tone class. Mandarin has four tone classes, namely, Yinping, Yangping, Shangsheng and Qusheng, "four tones" for short. The tone-values of four tones can be described with five-degree method.

(1) YinPing:

The highest pitched voice, the sound is high and flat, from 5 degree to 5 degree with no changes in the middle, which usually called the high/flat tone or 55 tone, such as "Chun Tian Hua Kai”.

(2)Yang Ping:

Moderate pitched voice, sound was raised from the tenor to the treble, that is, from 3 degree to 5 degree, which usually known as the high rising tone, moderate rising tone or 35 tone, such as "Ren Min Tuan Jie".

(3) Shangsheng: 
Pitched voice was lower at the beginning and then reduced to the minimum, and then rise to half high, that is, from 2 degree down to 1 degree and then rose to 4 degree. If the tone is from low to high, which often regarded as falling rising tone or 214 tone, such as "Yong Jiu You Hao". When one tone connected with the other tones, one should read the change tones. First, half - tone was read, and tone in the Yin Ping front should be read with half tone, from 214 tone to 21 tone or 211 tone, which is falling, because the beginning sound is low. In addition, to read straightly like the same tone, from 214 tone to 24 tone or 35 tone

(4)Qusheng:

Pitched voice was from high to low (or fully drop), since then the pitch was decreased, representing the five-degree method. It was reduced from 5 to 1 , and regarded as 51 tone. In order to relax the tight of vocal cord completely, sound was from high to low, with the shortest length.

\section{The characteristics and functions of Japanese tones}

First of all, it should be noted that the words' pronunciation in Japanese is relatively simple, in addition to Youon, each of pseudonym was a syllable section (note: this includes Chouon, Hatsuon and Sokuon). Pseudonyms are needed to be read one by one without missing any pseudonym, including Chouon, Hatsuon and Sokuon. For example, o, ka, a, sa, and n are 5 syllables (syllable section), and they have Chouon and Hatsuon. And sa, yo, tsu and to have three syllable sections, and they are Youon and Sokuon. Correct pronunciation should be made in order to remember them accurately. As little change in the pronunciation will result in the changes in meaning, or even become a completely different word. So how to grasp the tone? This depends on the core of the tone. For example, in Chinese, we have mā, má, mă , mà, while in Japanese, a me (rain), a me (maltose), ha re (chopsticks), ha re (beginning)

Although the languages spoken by Japanese and Chinese people are completely different, the Japanese writing system comes from Chinese. Chinese writing was emerged in fifteenth century and then introduced to Japan in sixth Century. The body of Chinese writing has been added. Japanese still remains many local dialects. Under the influence of mass media such as television and movies, Tokyo dialect is a basically regarded as standard Japanese which has been gradually extended to the whole country,

The "four tones" of Chinese are embodied in the inner part of a syllable, while in Japanese. There is no change in the tone of a syllable. The tone of Japanese is reflected in syllable and syllable, that is, the height of a particular syllable can only be reflected by the comparison of the adjacent syllables.

Chinese tone: Yin Ping (from high to high, 55) Yang Ping (from low to high, 45); rise (from high, to low and then high, 214); Qusheng (from low to high, 51).

Japanese tone: flat type, (from low to high and high), is equivalent to the begining at tune 2, and then tune 1. High type at the beginning, (from high to low and low), is equivalent to the beginning at tune 4, and then keep low. Moderate high type, (moderate high and then low), is equivalent to the beginning at tune 2, keep tune 1 in high-pitched voice, and then turn to tune 4, and then keep low in the end. Low type in the end, similar to the flat type, but it needs to lower down when follows with auxiliary word. Therefore, there was no tune 3 in the Japanese.

The tone in Japanese is high or low type, from high to low or from low to high. A pseudonym represents a syllable, including pseudonyms that represent unvoiced sound, voiced sound and half-voiced sound, Sokuon, Hatsuon and Chouon , but it does not include "ya”, "yu" and "yo" in the Youon, which means that the Youon was considered as one syllable such as "ki" and "yu" have one syllable, but not two syllables. However, "ki yu u" and "ku u" have two syllables in Youon. For 
example, "a” and “ me” are two syllables, but if they are read from low to high means "maltose”, while if they are read from high to low then means "rain"

Type: Regardless of the number of syllables of the words (at least 1 syllable), starting from the low syllable, and then raise the second syllable until last syllable without decline.

- Chair (i su)

- Mother (okaasann)

- Pencil (ennpitsu)

- Hiking (ennsoku)

- Money (kane)

- Clothes (kimono)

- Medicine (kusuri)

Type: Regardless of the number of syllables of the words (at least 1 syllable), starting from high syllable, and then down in the second syllable until the end without rising.

- Greetings (aisatsu)

- Line (ito)

- Sea (umi)

- Writing (kaku)

- Monkey (saru)

Type: Regardless of the number of syllables of the words (at least 2 syllables), starting from the low syllable, raise the second syllable, and then decrease in the third syllable until the end without rising.

- Red (akai)

- Blue (aoi)

- Home (ie)

- Get up (okiru)

- Paper (kami)

- White (shiroi)

Type: Regardless of the number of syllables of the words (at least 3 syllables), starting from the low syllable and raise the second syllable, then keep in the third syllable, finally, decrease in the fourth syllable until the end without raise.

- Cheerful (ureshii)

- Sing (utau)

- Women (onna)

- Man (otoko)

- Coffee (koohii)

Type; Regardless of the number of syllables of the words (at least 4 syllables), starting from the low syllable and raise the second syllable to the fourth syllable, and then decrease in the fifth syllable until the end without raise.

Stand up (tachiagaru)

Type: Regardless of the number of syllables of the words (at least 5 syllables), starting from the low syllable and raise the second syllable to the fifth syllable, and then decrease in the sixth syllable until the end without raise..

Mobile phone (keitaidennwa)

Type: Regardless of the number of syllables of the words (at least 6 syllables), starting from the low syllable and raise the second syllable to the sixth syllable, and then decrease in the seventh syllable until the end without raise.. 
It can be concluded that: in addition to "Type starting from high syllable and then decrease in the second syllable until the end without raise, the others are starting from the low syllable and raise in the second which keep to number of syllables, and then decrease to the end without raise". That is to say, pseudonyms behind the number of syllables are the position where the pronunciation declines.

\section{The function of the Japanese tone}

Boundary function was used to divide the boundary of semantic units (words or phrases). According to the rules of Japanese language, a pitch would not raise up when it fall down in the inner of semantic unit. Therefore, a pitch rises from low to high indicating a new starting of semantic unit. (tone changes at the end of the sentence is not included).

For example: ame (rain/sugar), hashi (beginning/chopstick), aki (autumn/)

In a word, once the tone is lowered it will not rise again. In Chinese the role of the tones is very important to distinguish the meaning of words.

But the proportion of words that can be distinguished by tones is small in Japanese language, such as words like "rain", or "chopsticks", "bridge". The main function of the Japanese tone is to divide the boundary of the semantic unit. However, although the semantic function of Japanese tone is weak, it does not mean that those foreigners who learn Japanese cannot master it. In phonological practice,

Japanese tones are important not only for distinguishing the meaning by listening sound, but also important for the leaner's Japanese mimicry (ie. whether it sounds like Japanese), learning Japanese quasi degree (whether it sounds like Japanese). For example, "a me" have two syllables, if you them from low to high means "sugar”, while it means "rain" when you read from high to low.

\section{The characteristics of Japanese tones}

First, in a word or phrase, the high pitches were focused on the same place. In other words, low syllable couldn't appear among high syllables. Second, the first syllable is different from the second syllable. If the first syllable was low, then the second syllable should be raised.

In addition, although the tone of Japanese does not have a significant impact on the meaning of words as Chinese dose, some words can also express different meanings due to different tones. We should pay special attention to this.

For example:

(1) a me (sugar)

a me (rain) (1)

(2) ka ki (persimmon) (O)

(3) u mi (suppuration) (2)

ka ki (summer) (1)

u mi (sea) (1)

(4) i shi (stone) (2)

i shi (will) (1)

(5) ha shi (bridge) (2)

ha shi (chopsticks) (1)

\section{Types of Japanese tone}

Refers to the first syllable is low, followed by high syllable in tone, and the tone should always be high enough to be followed by the auxiliary word.

High in the beginning, high in the middle and high in the end

High in the beginning: the first syllable is high, followed by low syllables. The tone is always 
low to the last auxiliary.

High in the middle: the first syllable is low, then starting to rise in the second syllable, and it falls down after the second syllable.

High in the end: the first syllable is low, then starting to rise in the second syllable til the end of the last syllable (down in the follow-up auxiliary). Some dictionaries using the number of 2, 3, 4, 5 and 6 to represent the type of "high in the end", and the number refers to the number of tone.

\section{Conclusions}

It can be concluded that in addition to Type (1) which means starting from the high syllable, and decrease in the second syllable until the end without raising, the others are all starting from the low syllable, and then starting to raise in the second syllable, keeping with the number of syllable, and then decrease to the last syllable without raising. That is to say, pronunciation behind the number of syllable is the place where the pronunciation drops.

In the actual process of learning words, there will be the following problems:

First, sometimes, there will be a word indicates two tones, indicating that this word has two pronunciations. Generally speaking, we can read the front mark or the latter mark.

Second, sometimes, a word is a compound word, which is made up of 2 words. So, there should be two marks in the tones, with "+" in the middle, which will appear the bimodal type. Because compound word is easy to identify, so there is no need to worry about bimodal type.

Third, the importance of the tone core lies in the correct pronunciation. But a variety of feelings might be mixed in the speech, such as: surprise, anger, doubt, anxiety, etc, which may influence the change in tone. Under normal circumstances, it will be fine as long as you make the correct pronunciation.

Forth, there are several comparative pronunciations need to remember: 1) those words like rain; sugar; bridge; chopsticks; come back; change, have the same pseudonyms while different in tones, representing different meanings. 2) in system of "ko so a to", ko le, so le, and a le in the beginning, middle, or end are all Type, while "dore" is regarded as Type in interrogative words, not only in the possessive pronoun, but also in direction words, conjuncted words and adverbs. 3) most interrogative words are belonged to Type, such as "nani”, “dare”, “ikutsu” etc.

Fifth, in the study you will find such a situation: if the word has three syllables, marked with, and some marked with Type. Of course, words have four syllables were marked with Type or Type; even some words have three syllables might mark with Type and Type. Words have four syllables were marked with Type and Type. After observation. words with three syllables were marked with Type , indicating that the follow-up auxiliary words are needed to be decreased when it was added. If it was marked with Type, indicating that follow-up auxiliary words are needed to be pronounced high without decline, which is the same as in four syllables.

If the words are marked with Type and Type, then two pronunciation are acceptable.

\section{References}

[1] Liu Chang; Literal or Free Translation in English-Chinese Film Translation-A Case Study of Pride and Prejudice[J] ; overseas English; 201108.

[2] Luo Xuelin; Japanese irony cognition and understanding of [D]; Guangdong University of Foreign Studies; 2006.

[3] He Gongxing; Japanese; Japanese Chinese names tone rule [J]; technology information; 2011 17 . 
[4] Genben Huang; [A] fluctuation of Japanese general

interrogative sentence; Ninth China phonetic conference [C]; 2010

[5] Yu Dongmei; Sino Japanese homographs Chinese characters words research [D]; Jilin University; 2012

[6] Zhang Li; “I am a cat”in the Japanese passive expression and translation [D]; Jilin University; 2012

[7] Long Juyang; Study on Chinese Japanese learners' learning concept [D]; Dalian University of Technology; 2010 